\title{
Modal analysis and harmonic response analysis of crane arm of medium and small sized automobile crane CAO Fu-yi ${ }^{1, a}$, CUI Meng-kai ${ }^{2, b^{*}}$ and LI Jin-long ${ }^{3, c}$ \\ ${ }^{1}$ Lankao County, Henan Province, Henan University of Science \& Technology, China ${ }^{2}$ Zhengding County, Shijiazhuang City, Hebei Province, Henan University of Science \& Technology, China \\ ${ }^{3}$ Xinmi City, Henan Province, china \\ acfy0908@sina.com, b1575587709@qq.com, c1542903038@qq.com
}

\section{Key word: Lifting arm; Vibration; modal analysis; Harmonic response analysis}

\begin{abstract}
Based on the Hypermesh software, the finite element modal analysis and harmonic response analysis of the truck crane boom are carried out. Through the modal analysis, the natural frequencies and modes of the first ten stages of the boom were obtained, so as to understand the effect of different frequency loads on the boom and the overall dynamic stiffness of the boom under various working conditions. This provides a theoretical basis for the structural design or further optimization of the boom design. By harmonic response analysis, the frequency of the three kinds of conditions of the displacement response curve, the most easily analyzed boom resonance frequency, the basis for subsequent analysis.
\end{abstract}

\section{Introduction}

In the traditional design of a crane boom, the design of jib, especially for medium and small tonnage, usually adopts the traditional mechanics method, the calculation is complicated and the calculation accuracy is low. Only the strength of several dangerous cross sections is taken into consideration in calculating, and the safety factor selection guarantee Its strength and reliability, thus failing to fully consider the impact and vibration problems caused by the dynamic load during the operation of the boom. Therefore, the dynamic analysis of the boom is very necessary[1-2].

Based on the static analysis of crane boom of a small and medium sized truck crane, finite element software Hypermesh is used to perform modal analysis and harmonic response analysis of the whole boom under various operating conditions of the boom. Modal analysis to extract the modal shapes and frequencies that affect the performance of the boom to understand the impact of different frequency load on the overall boom and the overall dynamic stiffness of the boom under various conditions, to provide a theoretical basis for the design and transformation. Harmonic response analysis is the response of a mechanical structure at a specific frequency to withstand dynamic loads that vary sinusoidally with time and help designers to verify that the structure can overcome resonance, fatigue and other effects under forced vibration.

\section{Modal analysis}

In structural dynamics analysis of boom, modal analysis theory is the basis. Modal analysis technology in engineering practice is widely used to evaluate the dynamic characteristics of boom structure system. By modal analysis can be obtained the natural frequencies and mode shapes of the boom system. When the boom is designed and improved, the natural frequency of the structure is avoided by the external excitation frequency. And through the modal analysis of the boom, the distribution of the overall bending stiffness and torsional stiffness of the boom system can be intuitively understood[3-4].

In the calculation of crane boom vibration, natural frequency and mode shape are the inherent characteristics of the boom, which is only related to the stiffness and mass distribution of the 
structure. Analysis of the relationship between the natural frequency of the boom and the mode shape helps to improve the structure of the boom[5].

\section{1 finite element model}

The QY10 crane studied in this paper uses a box-type hydraulic telescopic boom with a total of five knots.

Usually QY10 truck crane boom at work there are three conditions, the three conditions shown in Table 1:

Table 1. boom conditions

\begin{tabular}{cccc}
\hline condition & boom length $/ \mathrm{m}$ & the maximum weight $/ \mathrm{t}$ & work rate $/ \mathrm{m}$ \\
\hline basic arm condition & 7.98 & 10 & 3.5 \\
\hline intermediate arm condition & 13.0 & 5.6 & 4.0 \\
\hline full extension arm condition & 30.40 & 1.4 & 5.5 \\
\hline
\end{tabular}

Among them, the characteristic parameters of the materials used for the boom are shown in Table 2.

Table 2. boom material properties parameters

\begin{tabular}{llll}
\hline material & Elastic Modulus/MPa & Poisson's ratio & density/kg $\bullet \mathrm{m}-3 \quad$ Yield Strength/MPa \\
\hline
\end{tabular}

\begin{tabular}{lllll}
\hline $16 \mathrm{Mn}$ & $2.1 \times 105$ & 0.3 & 7850 & 590 \\
\hline
\end{tabular}

Meshing: In the process of establishing the finite element model of boom, the mesh is divided by tetrahedrons and trihedrons, and the mesh is refined at the transition fillet of the boom shaft to obtain 52169 units. 95682 nodes, the boom finite element model is shown in Fig.1.

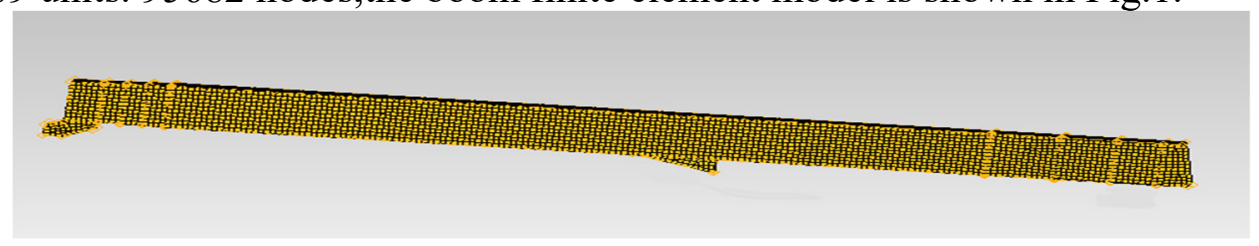

Fig.1.boom finite element model

\subsection{Solution and result analysis}

The modal analysis will be extended to the tenth order due to the natural frequency of each mode of vibration may cause the boom to break down in the form of resonance and so on. The relationship between natural frequency and mode shape of boom mode analysis obtained by finite element analysis is shown in Table 3. The deformation of the first four modes of the boom, as showed in Fig.2-4.

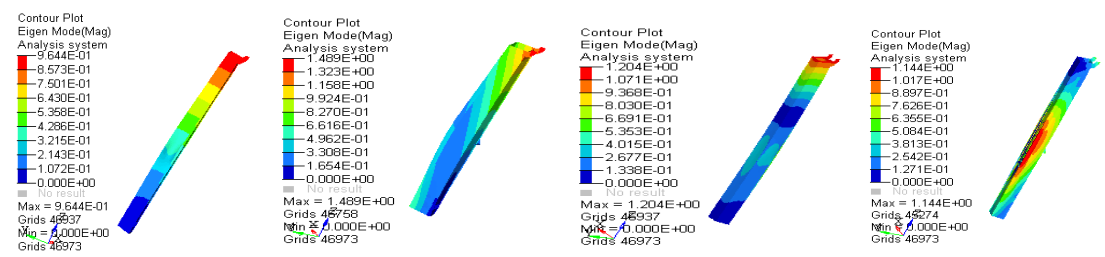

Fig.2.basic arm conditions mode shape diagram 


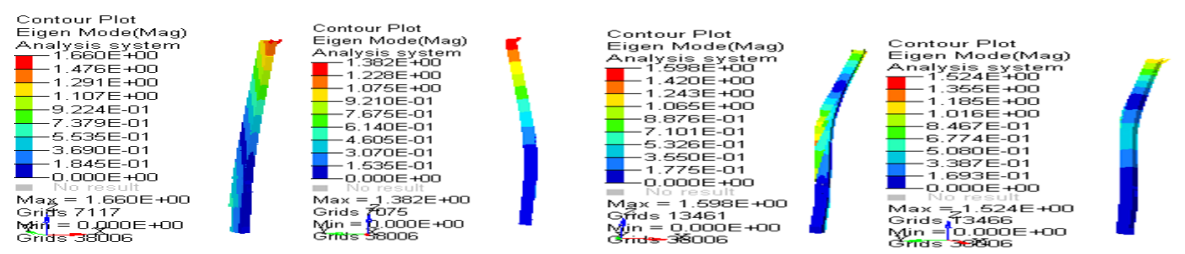

Fig.3.intermediate arm conditions mode shape diagram

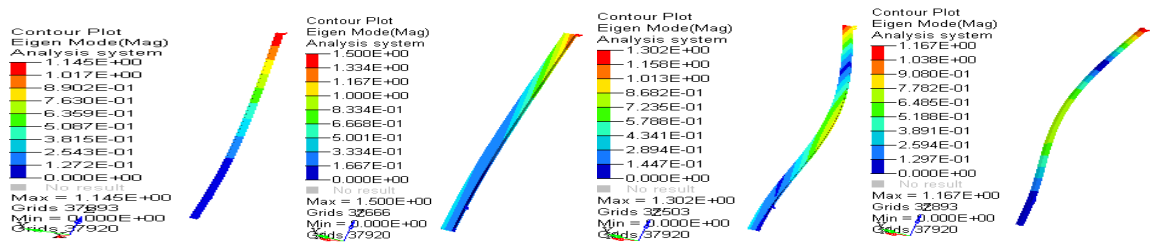

Fig.4.full extension arm conditions mode shape diagram

Table 3. parts of the frequency and mode description of modal analysis

\begin{tabular}{|c|c|c|c|c|c|c|}
\hline & \multicolumn{2}{|c|}{ basic arm condition } & \multicolumn{2}{|c|}{ intermediate arm condition } & \multicolumn{2}{|c|}{ full extension arm condition } \\
\hline & $\begin{array}{l}\text { frequen } \\
\text { cy }\end{array}$ & $\begin{array}{c}\text { Mode shape } \\
\text { description }\end{array}$ & $\begin{array}{c}\text { frequenc } \\
y\end{array}$ & $\begin{array}{l}\text { Mode shape } \\
\text { description }\end{array}$ & frequency & $\begin{array}{c}\text { Mode shape } \\
\text { description }\end{array}$ \\
\hline level one & 4.613 & $\begin{array}{l}\text { Vertical } \\
\text { bending mode }\end{array}$ & $\begin{array}{l}1.15424 \\
9\end{array}$ & $\begin{array}{l}\text { Lateral } \\
\text { bending mode }\end{array}$ & 0.65282 & $\begin{array}{c}\text { Lateral bending } \\
\text { mode }\end{array}$ \\
\hline $\begin{array}{l}\text { level } \\
\text { two }\end{array}$ & 5.8592 & $\begin{array}{l}\text { Lateral bending } \\
\text { mode }\end{array}$ & $\begin{array}{l}3.17029 \\
8\end{array}$ & $\begin{array}{l}\text { Vertical } \\
\text { bending mode }\end{array}$ & 1.16998 & $\begin{array}{c}\text { Vertical bending } \\
\text { mode }\end{array}$ \\
\hline level ten & 93.837 & $\begin{array}{l}\text { Similar to the } \\
\text { lateral sine } \\
\text { wave swing and } \\
\text { accompanied by } \\
\text { a twisting } \\
\text { swing }\end{array}$ & 51.423 & $\begin{array}{l}\text { Similar to do } \\
\text { vertical sine } \\
\text { wave swing }\end{array}$ & 30.4227 & $\begin{array}{l}\text { Similar to the } \\
\text { vertical sine wave } \\
\text { swing and } \\
\text { accompanied by a } \\
\text { twisting swing }\end{array}$ \\
\hline
\end{tabular}

Can be seen from the boom mode shape diagram, the boom mainly performs horizontal, vertical and torsional vibration when subjected to external excitation, and as the model order increases, the boom shape of the boom more and more complex. The basic arm operating condition showed obvious twisting at the 6th order. Both the long-arm and full-arm operating conditions were obviously reversed at the 8th order, which indicated that the anti-torsional ability of the boom structure is strong.It can be seen from the vibration modes of the boom of the basic boom that the vibration frequency of the boom in the swivel plane is always higher than the natural frequency of the boom in the luffing plane under the condition of the basic arm. This shows that the stiffness of the boom in the swivel plane is greater than Stiffness in the luffing plane. It can be seen from the vibration modes of the boom with full-boom and full-boom that the vibration frequency of the boom in the swivel plane is always higher than that of the boom in the luffing plane The low frequency indicates that the stiffness of the boom in the swivel plane is less than the stiffness in the swivel plane. Therefore, measures should be taken to strengthen the sidewall stiffness of the boom, such as adding a stiffener. The difference between the natural frequencies of the adjacent two stages of the boom in the low-order modes is small, so the resonance effect tends to occur.

It can be seen from the analysis that the maximum deformation point of the boom in the first 4 modes is concentrated at the boom head and most of the deformation of the boom in the latter 6 modes is concentrated at the junction of arm ends of every two sections. The reason for this is that as the external excitation frequency increases, the boom is gradually presented to the local vibration mode of vibration.

\section{Harmonic response analysis}

In the modal analysis, the low-order mode has the most influence, the high-order mode has less influence, and the higher the mode order, the smaller the impact[6]. So in this paper, we take the first ten frequencies of mode analysis as the frequency range of harmonic response analysis, that is, the working condition of basic arm is $0 \sim 90 \mathrm{~Hz}$, the working condition of middle and long arm is 0 
$\sim 40 \mathrm{~Hz}$, the working condition of full arm is $0 \sim 25 \mathrm{~Hz}$, Modal superposition method to calculate the arm end displacement response frequency curve of each condition shown in Fig.5.
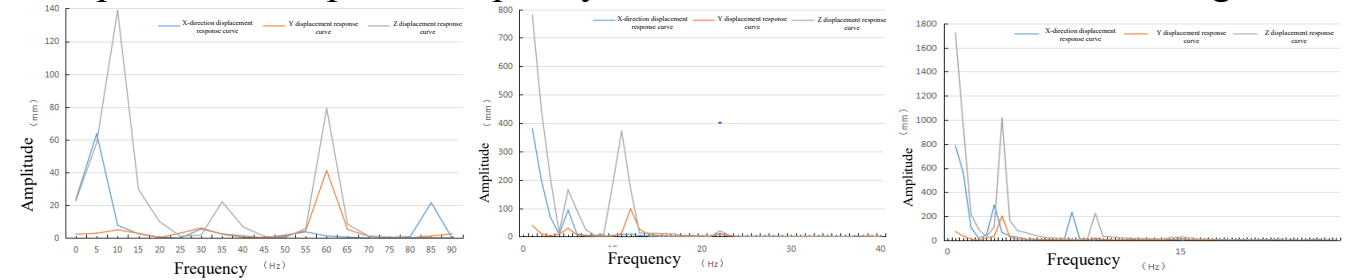

Fig.5.displacement frequency response curve

It can be seen from the response curve that the boom amplitude reaches the maximum at the frequencies of $6.925 \mathrm{~Hz}, 62.251 \mathrm{~Hz}$ and $10.366 \mathrm{~Hz}$ respectively in the $\mathrm{X}, \mathrm{Y}$ and $\mathrm{Z}$ directions under the condition of the basic arm, which are respectively $69.8 \mathrm{~mm}, 39.1 \mathrm{~mm}$ and $125.16 \mathrm{~mm} . Z$ direction produces another larger value of $69.879 \mathrm{~mm}$ at $62.7251 \mathrm{~Hz}$, which is close to the seventh natural frequency of the boom. In the middle and long arm conditions, the boom amplitude reached the maximum at frequencies of $1 \mathrm{~Hz}, 11 \mathrm{~Hz}$ and $1 \mathrm{~Hz}$ respectively at $378.95 \mathrm{~mm}, 90.21 \mathrm{~mm}$ and $789.15 \mathrm{~mm}$ along the $\mathrm{X}, \mathrm{Y}$ and $\mathrm{Z}$ directions respectively at $5 \mathrm{~Hz}$ for $\mathrm{X}, 1 \mathrm{~Hz}$ for $\mathrm{Y}$ and $\mathrm{Z}$ Another larger value is generated at $11 \mathrm{~Hz}$. The full boom boom boom along the $\mathrm{X}, \mathrm{Y}, \mathrm{Z}$ direction at the frequencies of $0.6 \mathrm{~Hz}, 4 \mathrm{~Hz}$ and $0.6 \mathrm{~Hz}$ maximum amplitude, respectively $791.32 \mathrm{~mm}, 198.95 \mathrm{~mm}$ and $1786.5 \mathrm{~mm}, \mathrm{X}$ to $3 \mathrm{~Hz}, 7.5 \mathrm{~Hz}, \mathrm{Z}$ An additional larger point is generated at $1 \mathrm{~Hz}, 3.5 \mathrm{~Hz}$. The actual work should be taken to avoid external excitation frequency falls in the vicinity of the above frequency.

From the above analysis can draw the following conclusions: 1) The maximum displacement peak in the $\mathrm{X}, \mathrm{Z}$ forward modes indicates that the first several modes have the largest horizontal and displacement of the boom. 2) The displacement in the vertical direction ( $Z$ direction) is the largest, which has a great influence on the dynamic performance of the structure and the smallest displacement in the axial direction (Y direction), which has little influence on the dynamic performance of the structure, which is related to the loading and the structural rigidity.

\section{Conclusion}

(1) Through the modal analysis of the boom, it can be seen that the difference of the natural frequency in the low-order mode is small, the resonance effect is easy to occur and the bending fatigue crack appears, which should be paid attention to in the structure design and the processing technology of the boom .

(2) Through the analysis of the harmonic response of the boom, the resonance frequency, resonance amplitude and principal vibration direction of the boom are obtained. It is concluded that the largest part of the resonance amplitude of the boom is in the boom head and the next is the arm connection. It provides theoretical support for the optimization design of follow-up boom.

\section{Acknowledgement}

This work was financially supported by Henan Province Science and Technology Research Project funded projects (172102210252).

\section{References:}

[1] Wen Hao. Crane Machinery [M]. Machinery Industry Press, 2013,9: 1-94.

[2] Wu Xiao.SQT160 three-arm railway crane finite element analysis and local stress calculation method [J]. Hoisting and transport machinery.1998 (3): 3-6.

[3] CHEN Hao, GUO Long-long, ZHAO Deng-yang et al. Static and modal analysis of crane boom on offshore platform [J].Petroleum Machinery, 2013,41 (10): 69-71. 
[4] WANG Ling-juan, ZHANG Li. Harmonic response analysis of tower crane jib mode [J]. Modern Manufacturing Engineering, 2017, (8): 137-140.

[5] Li Chuanlong.SQ5 crane finite element analysis [D]. Harbin University of Science and Technology, 2013.

[6] Miao Qiang. Elliptical telescopic boom dynamic characteristics and lightweight design [D]. Southwest Jiaotong University, 2015. 